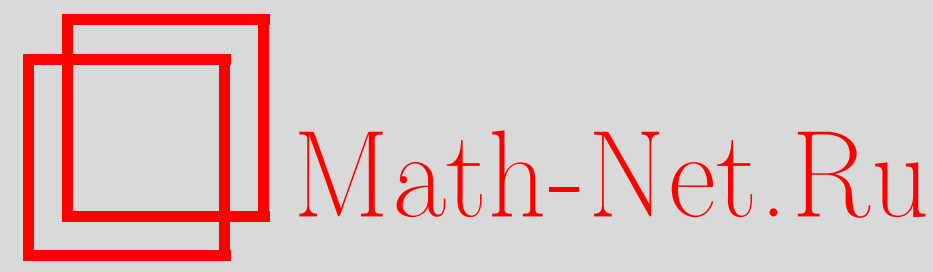

С. Е. Пастухова, О характере распределения поля температур в перфорированном теле с заданным его значением на внешней границе в условиях теплообмена на границе полостей по закону Ньютона, Матем. сб., 1996, том 187, номер 6, 85-96

DOI: https://doi.org/10.4213/sm138

Использование Общероссийского математического портала Math-Net.Ru подразумевает, что вы прочитали и согласны с пользовательским соглашением http://www . mathnet.ru/rus/agreement

Параметры загрузки:

IP : 54.210 .77 .194

26 апреля 2023 г., 16:54:59 
УДК 517.953

\author{
С.Е. Пастухова
}

\title{
О характере распределения поля температур в перфорированном теле с заданным его значением на внешней границе в условиях теплообмена на границе полостей по закону Ньютона
}

При $\varepsilon \in(0,1)$ пусть $\Omega_{\varepsilon}=\Omega \cap \varepsilon \omega$, где $\Omega \subset \mathbb{R}^{d}$ - ограниченная область, $\varepsilon \omega$ - множество, полученное сжатием в $\varepsilon^{-1}$ раз из $\omega$ - неограниченной области, имеющей 1-периодическую структуру, при этом множество $\mathbb{R}^{d} \backslash \omega$-дисперсно. Тогда $\partial \Omega_{\varepsilon}=\Gamma_{\varepsilon} \cup S_{\varepsilon}$, где $\Gamma_{\varepsilon}-$ внешняя граница $\Omega_{\varepsilon}, S_{\varepsilon}-$ граница полостей, лежащих в $\Omega_{\varepsilon}$.

Изучается эффект экспоненциально затухающего при $\varepsilon \rightarrow 0$ влияния ненулевого температурного режима, установленного на $\Gamma_{\varepsilon}$, на распределение температуры внутри изотропного тела, занимающего область $\Omega_{\varepsilon}$, при условии, что теплообмен на границе $S_{\varepsilon}$ со средой, заполняющей полости тела, осуществляется по закону Ньютона с коэффициентом пропорциональности $a_{\varepsilon}(x)=a(x / \varepsilon)$, где $a(y)$ - 1-периодическая функция, определенная на $\partial \omega$, такая, что $\int_{S} a(y) d s>0$, если $S=\partial \omega \cap\left\{x \in \mathbb{R}^{d}:\left|x_{i}\right|<1 / 2, i=\overline{1, d}\right\}$.

Библиограбоя: 8 названий.

\section{Введение}

Пусть $\Omega$-некоторая гладкая ограниченная область в $\mathbb{R}^{d}, \omega \in \mathbb{R}^{d}$-неограниченная область с 1-периодической структурой. Предположим, что множество $\mathbb{R}^{d} \backslash \omega$ дисперсно, т.е. оно распадается на непересекающиеся компоненты, причем каждая из этих компонент есть гладкая замкнутая ограниченная область. Пусть в ячейке периодичности $Q=\left\{x \in \mathbb{R}^{d}:\left|x_{i}\right|<1 / 2\right\}$ содержится лишш конечное число этих компонент и они не выходят на границу $\partial Q$, т.е., если $P=\omega \cap Q$ и $S=\partial(Q \backslash P)$, то $\rho(S, \partial Q)>0$.

Для $\varepsilon>0$ определим $\varepsilon \omega=\left\{x \in \mathbb{R}^{d}: \varepsilon^{-1} x \in \omega\right\}$, тогда получим "перфорированную" область $\Omega_{\varepsilon}=\Omega \cap \varepsilon \omega$, если удалим из $\Omega$ дисперсное множество $\Omega \backslash \varepsilon \omega$. Чем меньше $\varepsilon$, тем больше полостей оказьвается внутри $\Omega_{\varepsilon}$. Граница такой сильно перфорированной области имеет вид: $\partial \Omega_{\varepsilon}=\Gamma_{\varepsilon} \cup S_{\varepsilon}$, где $\Gamma_{\varepsilon}=\partial \Omega \cap \varepsilon \omega, S_{\varepsilon}=\partial(\varepsilon \omega) \cap \Omega$. Будем называть $\Gamma_{\varepsilon}$ внешней границей $\Omega_{\varepsilon}$, а $S_{\varepsilon}$ - границей полостей области $\Omega_{\varepsilon}$. Пусть, далее, $n=\left(n_{1}, \ldots, n_{d}\right)$ - вектор единичной внешней нормали на $S_{\varepsilon}$.

Рассмотрим стационарное распределение поля температур $u_{\varepsilon}(x)$ внутри изотропного тела, которое занимает область $\Omega_{\varepsilon}$, при этом на внешней границе его $\Gamma_{\varepsilon}$ поддерживается заданное поле температур, а на границе полостей $S_{\varepsilon}$ осушествляется теплообмен трех различных типов: 
1) поток тепла из $\Omega_{\varepsilon}$ на $S_{\varepsilon}$ нулевой, т.е. $-\left(\nabla u_{\varepsilon}, n\right)=0$ на $S_{\varepsilon}$;

2 ) на границе $S_{\varepsilon}$ поддерживается всюду нулевой температурный режим, т.е. $u_{\varepsilon}=0$ на $S_{\varepsilon}$

3) теплообмен на границе $S_{\varepsilon}$ идет по закону Ньютона, т.е. поверхностная плотность потока тепла из $\Omega_{\varepsilon}$ через $S_{\varepsilon}$ пропорциональна температуре, установившейся на $S_{\varepsilon}:-\left(\nabla u_{\varepsilon}, n\right)=a_{\varepsilon}(x) u_{\varepsilon}$, и пусть коэффициент пропорциональности $a_{\varepsilon}(x)=a(x / \varepsilon)$, где $a(y)$ - гладкая 1-периодическая функция, определенная на $\partial \omega$.

Первые два случая хорошо изучены. В первом случае поле температур $u_{\varepsilon}(x)$ можно усреднять при $\varepsilon \rightarrow 0$ (см. [1], [2]): поле $u_{\varepsilon}(x)$ достаточно хорошо приближается полем температур $u_{0}(x)$, которое есть поле температур для однородного тела (без полостей), заполняющего область $\Omega$, с тем же значением температур на внешней границе $\partial \Omega$, но с измененным по сравнению с первоначальньм тензором теплопроводности, и формулы для нахождения этого нового усредненного тензора известны. Во втором случае поле температур $u_{\varepsilon}(x)$ будет экспоненциально сходиться к нулевому при $\varepsilon \rightarrow 0$ вне границы $\Gamma_{\varepsilon}$ (см. [3, с. 135]), если отсутствуют объемные источники тепла внутри $\Omega_{\varepsilon}$.

В меньшей степени изучен третий случай с теплообменом на границе полостей, идушим по закону Ньютона. Отметим работы $[1$, с. 151] и [3], где этот случай рассматривался при условии, что $\int_{S} a(y) d s=0$. Было установлено, что при этом условии поле температур при $\varepsilon \rightarrow 0$ можно усреднять, и процедура усреднения сходна с той, что существует в первом случае отсутствия теплообмена через границу $S_{\varepsilon}$.

Наконец, рассмотрим случай, когда для третьего типа теплообмена на границе $S_{\varepsilon}$ коэффициент $a_{\varepsilon}(x)=a(x / \varepsilon)$ удовлетворяет условию:

$$
\frac{1}{\operatorname{mes} S} \int_{S} a(y) d s=\text { const }>0
$$

В этом случае налицо отток тепла из $\Omega_{\varepsilon}$ через $S_{\varepsilon}$ в тех точках $x$, где температура $u_{\varepsilon}(x)>0$ и коэффициент $a_{\varepsilon}(x)>0$. Поэтому, учитывая условие $(1)$, естественно в этой ситуации ожидать такого же качественного распределения поля температур $u_{\varepsilon}(x)$ внутри $\Omega_{\varepsilon}$ при $\varepsilon \rightarrow 0$, как при нулевом режиме температур на границе $S_{\varepsilon}$, а именно: $u_{\varepsilon}(x)$ экспоненциально близко к нулю в интегральной метрике $H^{1}$ вне любой окрестности внешней границы $\Gamma_{\varepsilon}$, если отсутствуют объемные источники тепла внутри $\Omega_{\varepsilon}$. Но также естественно ожидать, что порядок убывания к нулю будет различным при этих двух различных типах теплообмена на гранище $S_{\varepsilon}$. Убывание к нулю при $\varepsilon \rightarrow 0$ будет более быстрое при нулевом температурном режиме на границе $S_{\varepsilon}$.

Приведенные выше качественные результаты для третьего типа теплообмена на границе полостей $S_{\varepsilon}$ с условием (1) точно сформулированы и доказаны в $\S 2$, в теореме 2 , основной для этой работы. Заметим, что интерес представляет даже частньй случай рассматриваемой задачи, когда коэффициент пропорциональности

$$
a_{\varepsilon}(x)=k=\text { const }>0,
$$


и распределение температуры дается решением смешанной задачи для эллиптического оператора с классическим краевым условием на границе $S_{\varepsilon}$ третьего типа. Только в этом частном случае результат можно получить технически значительно проще, чем при общем условии (1) (при условии $\left(1^{\prime}\right)$ нет надобности в оценках (5), (22), крайне необходимых при общем условии (1)).

Условие (1) предполагает возможньм помимо случая $\left(1^{\prime}\right)$ рассматривать и другой, часто встречающийся в граничных задачах с третьим краевым условием, случай:

$$
\left.a_{\varepsilon}(x)\right|_{S_{\varepsilon}} \geqslant 0, \quad a_{\varepsilon}(x) \not \equiv 0
$$

(так как $a(y)$ - гладкая, то неотрицательность $a(y)$ влечет автоматически выполнение $(1)$, если $a(y) \not \equiv 0)$. Отметим, что похожая смешанная задача стационарной теплопроводности рассматривалась также в [5], [6], где охватывался случай, когда теплообмен на границе полостей $S_{\varepsilon}$ идет по закону Ньютона в предположении $\left(1^{\prime}\right)$ или $\left(1^{\prime \prime}\right)$. Но, если в настоящей работе основная цель - продемонстрировать эффект экспоненциально затухающего влияния ненулевого температурного режима, установленного на внешней границе $\Gamma_{\varepsilon}$, на участки $\Omega_{\varepsilon}$, отстоящие от $\Gamma_{\varepsilon}$ на положительном расстоянии, в предположении, что отсутствуют объемные тепловые источники внутри $\Omega_{\varepsilon}$ (или их плотность равна нулю), то в [5], [6] обсуждались совсем другие аспекты изучаемой задачи. Более точно, в [5], [6] рассматривался случай, когда объемные источники тепла есть и они лежат строго на некотором удалении от внешней границы $\Gamma_{\varepsilon}$, т.е. правая часть в уравнении теплопроводности (плотность объемных тепловых источников) является функцией из класса $D(\Omega)$, а на внешней границе $\Gamma_{\varepsilon}$ поддерживается нулевая температура. При таких предположениях в [5], [6] в соответствии с известной методикой (см. [1], [2]) построены асимптотики $u_{\varepsilon}(x)$ по степеням $\varepsilon: u_{\varepsilon}(x) \sim \varepsilon u_{0}(x)+\varepsilon^{2} u_{1}(x, x / \varepsilon)+\cdots$. Интересно, что полученные в настоящей работе результаты можно приложить для обоснования асимптотических разложений $u_{\varepsilon}(x)$ из [5], [6] в случае, когда объемные источники тепла могут выходить на внешнюю границу $\Gamma_{\varepsilon}$ (т.е. правая часть в уравнении теплопроводности не финитна в $\Omega$ ). Подробно аналогичные приложения граничных эффектов к обоснованию асимптотических разложений в случае задачи Дирихле для уравнения теплопроводности в перфорированной области приведены в $[3$, гл. III].

Для изучения поставленной задачи при условии (1), даже в простейшем его частном случае $\left(1^{\prime}\right)$, требуются некоторые дополнительные результаты о соотношениях между различными интегральньпи формами и нормами в перфорированных областях. Часть из них приведена в 1 , отметим среди них особенно важные для настоящей работы оценки (4), (5). С помощью этих же вспомогательных результатов удается также доказать теорему сушествования и единственности стационарного распределения температур в перфорированном теле (тривиальную при условии $\left(1^{\prime}\right)$ или даже $\left(1^{\prime \prime}\right)$, но не тривиальную, если отказаться от условия неотрицательности коэффициента $a_{\varepsilon}(x)$ во всех точках $\left.S_{\varepsilon}\right)$ с указанньм выше граничньм теплообменом в предположении (1) - вопрос, остававшийся открытым, несмотря на то, что постановка задачи находится в традиционных рамках смешанной эллиптической задачи с краевым условием I и III типов на границе. 
§1. Необходимые обозначения. Вспомогательные утверждения о нормах и интегральных формах в перфорированной области $\Omega_{\varepsilon}$

Далее нормы в пространствах $L^{2}\left(\Omega_{\varepsilon}\right), L^{2}\left(S_{\varepsilon}\right)$ будем обозначать, соответственно, через $|\cdot|_{\varepsilon},|\cdot|_{\varepsilon}^{\prime}$, а скалярные произведения в $L^{2}\left(\Omega_{\varepsilon}\right), L^{2}\left(S_{\varepsilon}\right)$, соответственно, - через $(\cdot, \cdot)_{\varepsilon},(\cdot, \cdot)_{\varepsilon}^{\prime}$.

Пусть $V_{\varepsilon}=H^{1}\left(\Omega_{\varepsilon}, \Gamma_{\varepsilon}\right)$ - замькание $C_{0}^{\infty}(\Omega)$ в норме $H^{1}\left(\Omega_{\varepsilon}\right)$. Для функции $u \in H^{1}\left(\Omega_{\varepsilon}\right)$ пусть $|\nabla u|_{\varepsilon}^{2} \equiv\left(\frac{\partial}{\partial x_{i}} u, \frac{\partial}{\partial x_{i}} u\right)_{\varepsilon}$, здесь и далее по повторяюшимся индексам подразумевается суммирование от 1 до $d$.

Пусть $\langle w\rangle_{M}=\frac{1}{\operatorname{mes} M} \int_{M} w d s$ - интегральное среднее функции $w$ по множеству $M$, и, значит, условие (1) принимает вид: $\langle a\rangle_{S}=$ const $>0$. Через $C_{i}, c_{i}, i \in \mathbb{N}$, обозначаем далее константы, не зависящие от $\varepsilon$.

Лемма 1. При всех $\varepsilon$ достаточно мальх $\left(\varepsilon<\varepsilon^{0}=\operatorname{const}(\Omega, P)\right)$ для любъх функиий $u \in V_{\varepsilon}$ верны неравенства:

$$
\begin{aligned}
|u|_{\varepsilon} & \leqslant C_{1}|\nabla u|_{\varepsilon}, \\
|u|_{\varepsilon}^{2} & \leqslant C_{2}\left(\varepsilon^{-1}|u|_{\varepsilon}^{2}+\varepsilon|\nabla u|_{\varepsilon}^{2}\right), \\
|u|_{\varepsilon}^{2} & \leqslant C_{3}\left(\varepsilon|u|_{\varepsilon}^{\prime 2}+\varepsilon^{2}|\nabla u|_{\varepsilon}^{2}\right), \\
\left|\int_{S_{\varepsilon}} \gamma\left(\frac{x}{\varepsilon}\right) u^{2} d s\right| & \leqslant C_{4} \gamma_{0}\left(2|u|_{\varepsilon}|\nabla u|_{\varepsilon}+\varepsilon|\nabla u|_{\varepsilon}^{2}\right),
\end{aligned}
$$

где $\gamma(y) \in C^{\infty}\left(\partial \omega, \mathbb{R}^{1}\right)$ - 1-периодическая по всем переменныц такая, что $\langle\gamma\rangle_{S}=0 u \gamma_{0}=|\gamma|_{C(S)}, C_{i}=\operatorname{const}(\Omega, P), i=\overline{1,4}$.

Оценка (2) хорошо известна и неоднократно использовалась в работах по усреднению (ее доказательство см., например, в [3, гл. ІІІ]). Доказательство оценок (3), (5) дано в [7], [8]. Оценка (4) доказывается аналогичным способом с помощью техники продолжения (см. [2, с. 43-44], а также [3, гл. III]) с использованием соображений подобия на основании хорошо известного неравенства:

$$
|u(y)|_{L^{2}(P)}^{2} \leqslant C_{5}\left(|u(y)|_{L^{2}(S)}^{2}+\left(\frac{\partial u}{\partial y_{i}}, \frac{\partial u}{\partial y_{i}}\right)_{L^{2}(P)}\right)
$$

справедливого для любой функции $u(y) \in H^{1}(P)$, где $C_{5}=\operatorname{const}(P)$.

\section{§2. Точная постановка задачи.}

\section{Основные результаты и их доказательство}

Поскольку изучается распределение температуры в изотропном теле, то его тензор теплопроводности имеет вид: $K_{i j}=k_{0} \delta_{i j}$, где $k_{0}=$ const $>0$ - коэффициент удельной теплопроводности, и поэтому без ограничения обшности можно считать, что $k_{0} \equiv 1$. Тогда стационарное поле температур $u_{\varepsilon}(x)$ в области $\Omega_{\varepsilon}$ с заданным его значением на внешней границе $\Gamma_{\varepsilon}$ и при теплообмене на границе полостей $S_{\varepsilon}$, 
идущим по закону Ньютона в предположении (1), если объемная плотность источников тепла есть $f(x)$, задается решением следуюшей смешанной задачи для оператора Лапласа $\Delta$ :

$$
\left\{\begin{array}{l}
\Delta u_{\varepsilon}=-f \text { в } \Omega_{\varepsilon}, \\
u_{\varepsilon}=g \text { на } \Gamma_{\varepsilon}, \\
\frac{\partial}{\partial n} u_{\varepsilon}+a_{\varepsilon}(x) u_{\varepsilon}=0 \text { на } S_{\varepsilon} .
\end{array}\right.
$$

Эту смешанную задачу будем рассматривать в обобщенном смысле, предполагая заданными функции $f \in L^{2}\left(\Omega_{\varepsilon}\right), g \in H^{1}\left(\Omega_{\varepsilon}\right)$.

ОПРедЕлЕниЕ 1 . Функция $u \in H^{1}\left(\Omega_{\varepsilon}\right)$ - решение (обобщенное) задачи (7), если $u=g$ на $\Gamma_{\varepsilon}$ и выполняется равенство с любой функцией $v \in V_{\varepsilon}$ :

$$
\left(\frac{\partial}{\partial x_{i}} u, \frac{\partial}{\partial x_{i}} v\right)_{\varepsilon}+\left(a_{\varepsilon}(x) u, v\right)_{\varepsilon}^{\prime}=(f, v)_{\varepsilon} .
$$

Для дальнейшего удобно и другое (эквивалентное) определение обобщенного решения задачи (7).

ОПРеДЕЛЕНиЕ 2. Функция $u \in H^{1}\left(\Omega_{\varepsilon}\right)$ вида $u=g+w$, где $w \in V_{\varepsilon}$, является решением (обобшенным) задачи (7), если для $w$ выполняется равенство с любой функцией $v \in V_{\varepsilon}$ :

$$
\left(\frac{\partial}{\partial x_{i}} w, \frac{\partial}{\partial x_{i}} v\right)_{\varepsilon}+\left(a_{\varepsilon}(x) w, v\right)_{\varepsilon}^{\prime}=-\left(\frac{\partial}{\partial x_{i}} g, \frac{\partial}{\partial x_{i}} v\right)_{\varepsilon}-\left(a_{\varepsilon}(x) g, v\right)_{\varepsilon}^{\prime}+(f, v)_{\varepsilon} .
$$

Далее в пространстве $V_{\varepsilon}$ будем рассматривать норму $\|\cdot\|_{\varepsilon}$, определяемую соотношением: $\|u\|_{\varepsilon}^{2}=|\nabla u|_{\varepsilon}^{2}+|u|_{\varepsilon}^{\prime 2}$.

TEOPEMA 1. При всех $\varepsilon$ достаточно малъи $\left(\varepsilon<\varepsilon^{0}=\operatorname{const}(\Omega, P, a(y))\right)$ для любых $f \in L^{2}\left(\Omega_{\varepsilon}\right), g \in H^{1}\left(\Omega_{\varepsilon}\right)$ существует и единственно решение задачи (7), и оно удовлетворяет оценкам:

$$
\begin{aligned}
\|u\|_{\varepsilon} & \leqslant C_{6}\left(|f|_{\varepsilon}+\|g\|_{\varepsilon}\right), \\
|u|_{\varepsilon} & \left.\leqslant C_{7}\left(\sqrt{\varepsilon}\left(|f|_{\varepsilon}+\|g\|_{\varepsilon}\right)+|g|_{\varepsilon}\right)\right),
\end{aligned}
$$

әде

$$
C_{i}=\operatorname{const}(\Omega, P, a(y)), \quad i=6,7 .
$$

ДокАЗАТЕЛЬСтво. Для доказательства утверждения теоремы 1 воспользуемся теоремой Лакса-Мильграма (см. [2, с. 13]). Докажем, что билинейная форма $\mathscr{B}(w, v)$, определяемая левой частью равенства $(9)$, удовлетворяет всем необходимым условиям этой теоремы. В самом деле,

$$
\mathscr{B}(w, w) \geqslant|\nabla w|_{\varepsilon}^{2}+\langle a\rangle_{S}|w|_{S}^{2}-C_{4} a_{0}\left(2|w|_{\varepsilon}|\nabla w|_{\varepsilon}+\varepsilon|\nabla w|_{\varepsilon}^{2}\right)
$$


на основании неравенства (5), если положить $a_{0}=\left|a(y)-\langle a\rangle_{S}\right|_{C(S)}$. Применим неравенство $|\alpha \cdot \beta| \leqslant \frac{1}{2} \rho \alpha^{2}+\frac{1}{2} \rho^{-1} \beta^{2}$, справедливое для любого $\rho>0$, считая, что $\alpha=|\nabla w|_{\varepsilon}, \beta=|w|_{\varepsilon}$, тогда, продолжая оценку $\mathscr{B}(w, w)$ снизу, получаем:

$$
\mathscr{B}(w, w) \geqslant\langle a\rangle_{S}|w|_{\varepsilon}^{\prime 2}+\left(1-a_{0} C_{4} \varepsilon-a_{0} C_{4} \rho\right)|\nabla w|_{\varepsilon}^{2}-C_{4} a_{0} \rho^{-1}|w|_{\varepsilon}^{2} .
$$

Последние слагаемые в (12) оценим в силу неравенства (4). В итоге получим:

$$
\mathscr{B}(w, w) \geqslant \lambda_{1}(\varepsilon)|\nabla w|_{\varepsilon}^{2}+\lambda_{2}(\varepsilon)|w|_{\varepsilon}^{\prime 2},
$$

где

$$
\begin{aligned}
& \lambda_{1}(\varepsilon)=1-a_{0} C_{4} \rho-a_{0} C_{4} \varepsilon-C_{4} a_{0} \rho^{-1} C_{3} \varepsilon^{2} \\
& \lambda_{2}(\varepsilon)=\langle a\rangle_{S}-C_{4} a_{0} \rho^{-1} C_{3} \varepsilon .
\end{aligned}
$$

Положив сначала $\rho$ достаточно малым (например, $\rho \leqslant \frac{1}{2}\left(a_{0} C_{4}\right)^{-1}$ ), выбираем далее $\varepsilon$ достаточно малым $\left(\varepsilon<\varepsilon^{0}\right)$ так, чтобы были обеспечены следующие три неравенства:

$$
a_{0} C_{4} \varepsilon<\frac{1}{8}, \quad C_{4} a_{0} \rho^{-1} C_{3} \varepsilon^{2}<\frac{1}{8}, \quad C_{4} a_{0} \rho^{-1} C_{3} \varepsilon<\frac{1}{2}\langle a\rangle_{S} .
$$

В случае их выполнения имеем равномерную по $\varepsilon$ оценку при $\varepsilon<\varepsilon^{0}$ :

$$
\lambda_{1}(\varepsilon) \geqslant C_{8}, \quad \lambda_{2}(\varepsilon) \geqslant C_{8}, \quad C_{8}=\operatorname{const}(\Omega, P, a(y))=0 .
$$

И, значит, из (13), (14) вытекает оценка:

$$
\mathscr{B}(w, w) \geqslant C_{8}\|w\|_{\varepsilon}^{2}
$$

означающая положительную определенность формы $\mathscr{B}(w, w)$ в пространстве $V_{\varepsilon}$ относительно нормы $\|\cdot\|_{\varepsilon}$ при $\varepsilon<\varepsilon^{0}$.

Легко вьвести оценку:

$$
|\mathscr{B}(w, v)| \leqslant C_{9}\|w\|_{\varepsilon}\|v\|_{\varepsilon} .
$$

Если $\mathscr{L}(v)$ - линейный функционал, определяемьй правой частью равенства $(9)$, то, используя оценку (2), можно доказать, что

$$
|\mathscr{L}(v)| \leqslant C_{10}\left(\|g\|_{\varepsilon}+|f|_{\varepsilon}\right)\|v\|_{\varepsilon} .
$$

Из (15)-(17) в силу теоремы Лакса-Мильграмма вытекает существование единственного элемента $w \in V_{\varepsilon}$ такого, что $\mathscr{B}(w, v)=\mathscr{L}(v)$ при любых функциях $v \in V_{\varepsilon}$, при этом

$$
\|w\|_{\varepsilon} \leqslant C_{11}\left(\|g\|_{\varepsilon}+|f|_{\varepsilon}\right)
$$

и, значит, функция $u=g+w$ есть единственное решение задачи (7) и для нее вьполнена оценка (10). Оценка (11) есть следствие оценки (10), если учесть неравенство (4). Теорема 1 доказана.

Следующая теорема 2 показывает, что решение задачи (7) экспоненциально близко к нулю в интегральной метрике вне $\mathscr{O}$ - произвольной окрестности $\Gamma_{\varepsilon}$ в $\Omega_{\varepsilon}$, если supp $f \subset \mathscr{O}$. Эта теорема является аналогом леммы 8 из главы III [3], где рассматривалась подобная задача с нулевьп условием Дирихле на границе $S_{\varepsilon}$. 
Tеорема 2. Пусть $u_{\varepsilon}-$ решение задачи (7) с функиией $f \equiv 0$ вне $\mathscr{O}(\mathscr{O}-$ некоторая окрестность $\Gamma_{\varepsilon}$ в $\left.\Omega_{\varepsilon}\right)$. Тогда для любой области $\Omega^{\prime}$ такой, что $\bar{\Omega}^{\prime} \subset \Omega, \bar{\Omega}^{\prime} \cap \mathscr{O}=\varnothing$, при всех $\varepsilon$ достаточно мальх $\left(\varepsilon<\varepsilon^{0}\right)$ справедлива оченка:

$$
\left|u_{\varepsilon}\right|_{H^{1}\left(\Omega^{\prime} \cap \Omega_{\varepsilon}\right)}^{2} \leqslant C \exp \left(-\frac{\delta}{\sqrt{\varepsilon}}\right)\left(|g|_{H^{1}\left(\Omega_{\varepsilon}\right)}^{2}+|f|_{L^{2}\left(\Omega_{\varepsilon}\right)}^{2}\right),
$$

где $C, \delta$ - положительные константы, которые не зависят от $\varepsilon$, но зависят om $\Omega, \Omega^{\prime}, P, a(y)$.

СлеДСТвИЕ. Если $f \equiv 0$, то оценка (18) выполняется для любой области $\Omega^{\prime}$ такой, что $\bar{\Omega}^{\prime} \subset \Omega$.

Теорема 2 следует из одной экспоненциальной оценки для решения (7), которая будет приведена в теореме 3 . Прежде чем сформулировать теорему 3 , введем вспомогательные функцию и множество. Пусть $\tau(x)$ - гладкая финитная неотрицательная функция такая, что $\operatorname{supp} \tau \subset \Omega$ и для всех $x \in \operatorname{supp} \tau$ с некоторой константой $M>0$

$$
\left|\frac{\partial}{\partial x_{i}} \tau(x)\right| \leqslant M, \quad i=\overline{1, d}
$$

После фиксации функции $\tau(x)$ выберем $\varepsilon$ столь малым, чтобы нашлась область $\Omega_{1}$ такая, что $\bar{\Omega}_{1} \subset \Omega$ и $\Omega_{1}$ - множество внутренних точек множества $\bigcup_{z \in T_{\varepsilon}} \varepsilon(Q+z)$, где $T_{\varepsilon}$-некоторое подмножество из $\mathbb{Z}^{d}$, при этом граница $\partial \Omega_{1}$ лежит в окрестности $\partial \Omega$, где $\tau \equiv 0$. Положим, далее, $\Omega_{1} \cap \Omega_{\varepsilon}=\Omega_{1}^{\varepsilon}, S_{1}^{\varepsilon}=\left(\partial \Omega_{1}^{\varepsilon}\right) \backslash \partial \Omega_{1}$.

Для дальнейшего понадобятся также некоторые варианты оценок (4), (5) для области $\Omega_{1}^{\varepsilon}$ и функций $u \in H^{1}\left(\Omega_{\varepsilon}\right)$, не обращающихся в нуль на $\Gamma_{\varepsilon}$, которые приводятся в следующей лемме.

Лемма 2. Пусть функция $\gamma(y)$ такая же, как в лемме 1, с нормой $\gamma_{0}=$ $|\gamma(y)|_{C(S)}$, a $\tau(x)$ и $\Omega_{1}^{\varepsilon}-$ функиия и множество, определенные согласованныц между собой образом перед леммой 2. Тогда при всех достаточно малых $\varepsilon$ $\left(\varepsilon<\varepsilon^{0}\right)$ для любой функции $u \in H^{1}\left(\Omega_{\varepsilon}\right)$ выполняются оценки:

$$
\begin{aligned}
|u|_{L^{2}\left(\Omega_{1}^{\varepsilon}\right)}^{2} & \leqslant C_{5}\left(\varepsilon|u|_{L^{2}\left(S_{1}^{\varepsilon}\right)}^{2}+\varepsilon^{2}\left|\nabla_{x} u\right|_{L^{2}\left(\Omega_{1}^{\varepsilon}\right)}^{2}\right), \\
\left|\int_{S_{1}^{\varepsilon}} \gamma\left(\frac{x}{\varepsilon}\right) u^{2} d s\right| & \leqslant C_{12} \gamma_{0}\left(2|u|_{L^{2}\left(\Omega_{1}^{\varepsilon}\right)}\left|\nabla_{x} u\right|_{L^{2}\left(\Omega_{1}^{\varepsilon}\right)}+\varepsilon\left|\nabla_{x} u\right|_{L^{2}\left(\Omega_{1}^{\varepsilon}\right)}^{2}\right), \\
\left|\int_{S_{1}^{\varepsilon}} \gamma\left(\frac{x}{\varepsilon}\right) u^{2} d s\right| & \leqslant C_{13} \gamma_{0} \sqrt{\varepsilon}\left(|\nabla u|_{L^{2}\left(\Omega_{1}^{\varepsilon}\right)}^{2}+|u|_{L^{2}\left(S_{1}^{\varepsilon}\right)}^{2}\right), \\
\int_{\Omega_{1}^{\varepsilon}} u^{2} \psi_{\varepsilon} d x & \leqslant C_{14}\left(\varepsilon \int_{S_{1}^{\varepsilon}} u^{2} \psi_{\varepsilon} d s+\varepsilon^{2} \int_{\Omega_{1}^{\varepsilon}}|\nabla u|^{2} \psi_{\varepsilon} d x\right),
\end{aligned}
$$

где $C_{5}$ - константа из неравенства $(6), \psi_{\varepsilon}(x)=\exp (\mu \tau(x)), C_{i}=\operatorname{const}(P)$, $i=\overline{12,14}$, константа $\mu=\mu(\varepsilon)$ определяется соотношением:

$$
\mu=M^{-1} \sigma\left(C_{5} \varepsilon\right)^{-1 / 2},
$$

где $C_{5}, M$ - константы из оценок (6), (19), а $\sigma \in(0,1)$ - произвольный параметр, не зависящий от $\varepsilon$. 
ДоказАтЕльство. 1) Оценки (20), (21) доказываются аналогично, как оценки $(4),(5)$, соответственно, и даже проше, потому что в этом случае не надо пользоваться техникой продолжения функций $v \in V_{\varepsilon}$ во внешность области $\Omega_{\varepsilon}$, поскольку множества $S_{1}^{\varepsilon}, \Omega_{1}^{\varepsilon}$ целиком состоят из компонент гомотетичных $S$ и $P$, соответственно. Применяя неравенство $(20)$ к одному из сомножителей, стоящих в правой части (21), легко вывести и оценку (22).

2) Для доказательства (23) применим оценку (20) к функции $u \exp (\mu \tau / 2)$. Это дает:

$$
J \equiv \int_{\Omega_{1}^{\varepsilon}} u^{2} e^{\mu \tau} d x \leqslant C_{5} \varepsilon \int_{S_{1}^{\varepsilon}} u^{2} e^{\mu \tau} d s+C_{5} \varepsilon^{2} \int_{\Omega_{1}^{\varepsilon}}\left|\nabla\left(u e^{\mu \tau / 2}\right)\right|^{2} d x \equiv J_{1}+J_{2}
$$

Оценим слагаемое $J_{2}$ из (25). Для этого заметим, что

$$
\frac{\partial}{\partial x_{j}}\left(u e^{\mu \tau / 2}\right)=\left(\frac{\partial}{\partial x_{j}} u+\frac{1}{2} \mu \frac{\partial \tau}{\partial x_{j}} u\right) e^{\mu \tau / 2}, \quad j=\overline{1, d}
$$

Поэтому

$$
J_{2} \leqslant C_{5} \varepsilon^{2}\left(2 \int_{\Omega_{1}^{\varepsilon}}|\nabla u|^{2} e^{\mu \tau} d x+\frac{1}{2} \mu^{2} M^{2} d \int_{\Omega_{1}^{\varepsilon}} u^{2} e^{\mu \tau} d x\right)
$$

Далее всюду считаем, что $\varepsilon<1 / d$, тогда имея в виду соотношение $(24)$, получаем, учитывая, что $\sigma \in(0,1)$, оценку:

$$
C_{5} \varepsilon^{2} \frac{d}{2} \mu^{2} M^{2}=\frac{d}{2} \sigma^{2} \varepsilon \leqslant \frac{d}{2} \varepsilon \leqslant \frac{1}{2}
$$

пользуясь которой на основании последнего неравенства для $J_{2}$, выводим из (25) справедливость оценки (23). И лемма 2 доказана.

Теорема 3. Пусть $и$-решение задачи (7) с функцией $f \equiv 0$ на $\Omega_{1}^{\varepsilon}$, функиия $\tau(x)$ и множество $\Omega_{1}^{\varepsilon}$ определень перед леммой 2. Тогда при всех достаточно мальх $\varepsilon\left(\varepsilon<\varepsilon^{0}\right)$ имеет место оценка с константой $C_{15}$, не зависящ, ей от в:

$$
\begin{aligned}
\varepsilon^{-1} \int_{\Omega_{1}^{\varepsilon}} u^{2} \psi_{\varepsilon} d x+\int_{\Omega_{\varepsilon}}|\nabla u|^{2} \psi_{\varepsilon} d x & +\int_{S_{\varepsilon}} u^{2} \psi_{\varepsilon} d s \\
& \leqslant C_{15}\left(\int_{\Omega_{\varepsilon}}|\nabla u|^{2} d x+\int_{S_{\varepsilon}} u^{2} d s\right)
\end{aligned}
$$

әде весовая функиия $\psi_{\varepsilon}(x)$ определена в лемме 2 с достаточно малым параметром $\sigma \quad\left(\sigma<\sigma^{0}\right)$. 
ДоКАЗАТЕЛЬСТВо ТЕОРЕМЫ 3. Если $u$ - решение задачи (7), то с любой функцией $v \in V_{\varepsilon}$ выполняется интегральное равенство (8). Пусть в этом равенстве $v=(\exp (\mu \tau(x))-1) u$, где множитель $\mu=\mu(\varepsilon)$ определяется формулой $(24)$. Тогда это равенство можно переписать в следующем виде:

$$
\begin{aligned}
\int_{\Omega_{\varepsilon}}|\nabla u|^{2} e^{\mu \tau} & d x+\int_{S_{1}^{\varepsilon}} a_{\varepsilon}(x) u^{2} e^{\mu \tau} d s \\
& =\int_{S_{1}^{\varepsilon}} a_{\varepsilon}(x) u^{2} d s+\int_{\Omega_{\varepsilon}}|\nabla u|^{2} d x-\mu \int_{\Omega_{1}^{\varepsilon}} \frac{\partial u}{\partial x_{i}} \frac{\partial \tau}{\partial x_{i}} u e^{\mu \tau} d x
\end{aligned}
$$

В равенстве (27) в некоторых интегралах область интегрирования $\Omega_{\varepsilon}, S_{\varepsilon}$ заменена на $\Omega_{1}^{\varepsilon}, S_{1}^{\varepsilon}$, соответственно, что возможно в силу согласованного выбора функции $\tau(x)$ и области $\Omega_{1}^{\varepsilon}: \tau \equiv 0$ вне $\Omega_{1}^{\varepsilon}$ и, значит, $v \equiv 0$ вне $\Omega_{1}^{\varepsilon}$. По той же причине в (27) отсутствует слагаемое $(f, v)_{\varepsilon}$, так как оно оказьвается нулевым в силу предположений на функцию $f$.

Ввиду оценки (22), если ее применить с весовой функцией $\gamma(y)=a(y)-\langle a\rangle_{S}$ (и тогда окажется $\left.\gamma_{0}=|\gamma|_{C(S)}=\left|a(y)-\langle a\rangle_{S}\right|_{C(S)} \equiv a_{0}\right)$ к функции $u \exp (\mu \tau / 2)$, получаем:

$$
\begin{aligned}
\Delta_{1} & \equiv\left|\int_{S_{1}^{\varepsilon}} a\left(\frac{x}{\varepsilon}\right) u^{2} e^{\mu \tau} d s-\langle a\rangle_{S} \int_{S_{1}^{\varepsilon}} u^{2} e^{\mu \tau} d s\right| \\
& \leqslant C_{13} a_{0} \sqrt{\varepsilon}\left(\left|\nabla\left(u e^{\mu \tau / 2}\right)\right|_{L^{2}\left(\Omega_{1}^{\varepsilon}\right)}^{2}+\left|u e^{\mu \tau / 2}\right|_{L^{2}\left(S_{1}^{\varepsilon}\right)}^{2}\right) .
\end{aligned}
$$

В силу свойств функции $\tau(x)$, используя неравенство (23), имеем:

$$
\begin{aligned}
& \left|\nabla\left(u e^{\mu \tau / 2}\right)\right|_{L^{2}\left(\Omega_{1}^{\varepsilon}\right)}^{2} \\
& \quad \leqslant 2 \int_{\Omega_{1}^{\varepsilon}}|\nabla u|^{2} e^{\mu \tau} d x+\frac{d}{2} \mu^{2} M^{2} \int_{\Omega_{1}^{\varepsilon}} u^{2} e^{\mu \tau} d x \\
& \quad \leqslant\left(2+\frac{d}{2} \mu^{2} M^{2} C_{14} \varepsilon^{2}\right) \int_{\Omega_{1}^{\varepsilon}}|\nabla u|^{2} e^{\mu \tau} d x+\varepsilon \frac{d}{2} C_{14} \mu^{2} M^{2} \int_{S_{1}^{\varepsilon}} e^{\mu \tau} d s .
\end{aligned}
$$

Поэтому, если учесть точную зависимость множителя $\mu=\mu(\varepsilon)$ от параметров $\varepsilon$ и $\sigma \in(0,1)($ см. $(24))$, при достаточно малых $\left(\varepsilon<\varepsilon^{0}\right)$ две последние оценки вкупе дают следуюшее неравенство:

$$
\Delta_{1} \leqslant C_{16} \sqrt{\varepsilon}\left(\int_{\Omega_{1}^{\varepsilon}}|\nabla u|^{2} e^{\mu \tau} d x+\int_{S_{1}^{\varepsilon}} u^{2} e^{\mu \tau} d s\right)
$$

где $C_{16}$ - константа, не зависящая от $\varepsilon$ и $\sigma \in(0,1)$.

Оценив последнее слагаемое правой части в (27) по неравенству Коши-Буняковского, выводим из $(27),(28)$ в силу определения величины $\Delta_{1}$ основное для 
дальнейшего доказательства неравенство:

$$
\begin{aligned}
\int_{\Omega_{\varepsilon}}|\nabla u|^{2} e^{\mu \tau} d x+\langle a\rangle_{S} \int_{S_{1}^{\varepsilon}} u^{2} e^{\mu \tau} d s \\
\leqslant C_{17}\left(\int_{\Omega_{\varepsilon}}|\nabla u|^{2} d x+\int_{S_{1}^{\varepsilon}} u^{2} d s\right) \\
+C_{17} \sqrt{\varepsilon}\left(\int_{\Omega_{1}^{\varepsilon}}|\nabla u|^{2} e^{\mu \tau} d x+\int_{S_{1}^{\varepsilon}} u^{2} e^{\mu \tau} d s\right) \\
+C_{17} \mu M\left(\int_{\Omega_{1}^{\varepsilon}}|\nabla u|^{2} e^{\mu \tau} d x\right)^{1 / 2}\left(\int_{\Omega_{1}^{\varepsilon}} u^{2} e^{\mu \tau} d x\right)^{1 / 2} .
\end{aligned}
$$

Оценивая второй множитель в последнем слагаемом неравенства (29) в силу неравенства (23) и выбирая $\varepsilon$ достаточно малым, так чтобы $C_{17} \sqrt{\varepsilon}<\frac{1}{2}$, $C_{17} \sqrt{\varepsilon}<\frac{1}{2}\langle a\rangle_{S}$, выводим из (29) следующую оценку:

$$
\begin{gathered}
\int_{\Omega_{\varepsilon}}|\nabla u|^{2} e^{\mu \tau} d x+\int_{S_{1}^{\varepsilon}} u^{2} e^{\mu \tau} d s \\
\leqslant C_{18}\left(\int_{\Omega_{\varepsilon}}|\nabla u|^{2} d x+\int_{S_{1}^{\varepsilon}} u^{2} d s\right) \\
+C_{18} \mu M \sqrt{\varepsilon} \int_{S_{1}^{\varepsilon}} u^{2} e^{\mu \tau} d s \\
+C_{18} \mu M \varepsilon \int_{\Omega_{1}^{\varepsilon}}|\nabla u|^{2} e^{\mu \tau} d x
\end{gathered}
$$

где константа $C_{18}$ не зависит от $\varepsilon, \sigma$.

Если необходимо, уменњшим допустимые значения $\varepsilon$ так, чтобы

$$
C_{18} \mu M \varepsilon<\frac{1}{2}
$$

и с этого момента считаем достаточно мальм и параметр $\sigma$ так, чтобы (см. (24))

$$
C_{18} \mu M \sqrt{\varepsilon}=C_{18}\left(C_{5}\right)^{-1 / 2} \sigma<\frac{1}{2} .
$$

Тогда из (30)-(32) вытекает оценка:

$$
\int_{\Omega_{\varepsilon}}|\nabla u|^{2} e^{\mu \tau} d x+\int_{S_{1}^{\varepsilon}} u^{2} e^{\mu \tau} d s \leqslant C_{19}\left(\int_{\Omega_{\varepsilon}}|\nabla u|^{2} d x+\int_{S_{1}^{\varepsilon}} u^{2} d s\right),
$$

откуда, используя (23), получаем также:

$$
\begin{aligned}
\varepsilon^{-1} \int_{\Omega_{1}^{\varepsilon}} u^{2} e^{\mu \tau} d x+\int_{\Omega_{\varepsilon}}|\nabla u|^{2} e^{\mu \tau} d x+\int_{S_{1}^{\varepsilon}} u^{2} e^{\mu \tau} d s & \\
& \leqslant C_{20}\left(\int_{\Omega_{\varepsilon}}|\nabla u|^{2} d x+\int_{S_{1}^{\varepsilon}} u^{2} d s\right) .
\end{aligned}
$$

И в последнем неравенстве $S_{1}^{\varepsilon}$ можно заменить на $S_{\varepsilon}$, считая $C_{20} \geqslant 1$ (в противном случае заменим константу $C_{20}$ единичной константой), так как $\exp (\mu \tau) \equiv 1$ на $S_{\varepsilon} \backslash S_{1}^{\varepsilon}$. Учитывая это замечание, выводим отсюда оценку (26). И теорема 3 доказана. 
ДОКАЗАТЕЛЬСТВо ТЕОРЕМЫ 2. ДЛЯ фиксированной области $\Omega^{\prime}$ такой, что $\bar{\Omega}^{\prime} \subset \Omega, \bar{\Omega}^{\prime} \cap \overline{\mathscr{O}}=\varnothing$, найдем число $\rho=\rho\left(\Omega^{\prime}, \mathscr{O}\right)>0$ и возьмем в качестве промежуточной области между $\Omega$ и $\Omega^{\prime}$ область $\Omega_{1}$ - множество внутренних точек множества $\bigcup_{z \in T_{\varepsilon}} \varepsilon(Q+z)$, где $T_{\varepsilon}$ - некоторое подмножество $\mathbb{Z}^{d}$, так чтобы $\bar{\Omega}^{\prime} \subset \Omega_{1}$, $\bar{\Omega}_{1} \cap \mathscr{O}=\varnothing, \rho\left(\partial \Omega_{1}, \Omega^{\prime}\right)>\rho / 2$. Если $\varepsilon$ достаточно мало, то задача построения такого множества $\Omega_{1}$ выполнима. Пусть, далее, $\Omega_{1}^{\varepsilon}=\Omega_{1} \cap \Omega_{\varepsilon}$.

Пусть $\tau(x)$ - гладкая финитная неотрицательная функция со следующими свойствами: $\tau \equiv 1$ на $\Omega^{\prime}, \tau \equiv 0$ вне $\rho / 2$-окрестности множества $\Omega^{\prime},\left|\frac{\partial \tau(x)}{\partial x_{j}}\right| \leqslant$ $c_{1} \rho^{-1}=M$ для всех $j=\overline{1, d}, x \in \operatorname{supp} \tau$ с некоторой константой $c_{1}$.

Применим к решению задачи (7) последовательно оценки (26) (выбор множества $\Omega_{1}^{\varepsilon}$ и функции $\tau(x)$ согласован так же, как в теореме 3$),(10),(3)$, тогда получим цепочку неравенств:

$$
\begin{aligned}
\varepsilon^{-1} \int_{\Omega_{1}^{\varepsilon}} u^{2} \psi_{\varepsilon} d x+\int_{\Omega_{1}^{\varepsilon}}|\nabla u|^{2} \psi_{\varepsilon} d x & \leqslant C_{15}\|u\|_{\varepsilon}^{2} \leqslant C_{21}\left(\|g\|_{\varepsilon}^{2}+|f|_{\varepsilon}^{2}\right) \\
& \leqslant C_{22}\left(\varepsilon^{-1}|g|_{H^{1}\left(\Omega_{\varepsilon}\right)}^{2}+|f|_{L^{2}\left(\Omega_{\varepsilon}\right)}^{2}\right) .
\end{aligned}
$$

Предполагая $\varepsilon<1$ и вспоминая вид весовой функции $\psi_{\varepsilon}(x)$, определенной в лемме 2: $\psi_{\varepsilon}(x)=\exp \left(\frac{\delta_{1}}{\sqrt{\varepsilon}} \tau(x)\right)$, где $\delta_{1}$ - константа, не зависяшая от $\varepsilon$, а также используя свойство функции $\tau(x): \tau(x)=1$ на $\Omega^{\prime}$, выводим из (33), сужая область интегрирования в левой части до $\Omega^{\prime}$ :

$$
e^{\delta_{1} / \sqrt{\varepsilon}}|u|_{H^{1}\left(\Omega_{\varepsilon}^{\prime}\right)}^{2} \leqslant C_{22}\left(\varepsilon^{-1}|g|_{H^{1}\left(\Omega_{\varepsilon}\right)}^{2}+|f|_{L^{2}\left(\Omega_{\varepsilon}\right)}^{2}\right) .
$$

Если $\varepsilon^{-1} e^{-\delta_{1} /(2 \sqrt{\varepsilon})} \leqslant c_{0}$ для всех $\varepsilon \in(0,1)$, то из оценки (34) вытекает оценка (18) с $\delta=\delta_{1} / 2$ и константой $C=\max \left(c_{0} C_{22}, C_{22}\right)$. Теорема 2 доказана.

ЗАмЕчАниЕ 1. В аналогичной задаче стационарной теплопроводности для перфорированного тела с заданњым температурным режимом на внешней границе $\Gamma_{\varepsilon}$, не равньм тождественно нулю, и постоянным нулевым температурным режимом на границе полостей $S_{\varepsilon}$ получается аналогичная, как в теореме 2 , оценка решения, только в ней экспоненциальный множитель вида $\exp (-\delta / \varepsilon)$ (см. [3, с. 135]). Отсюда ясно, что поле температур убывает к нулю вне границы $\Gamma_{\varepsilon}$ при отсутствии объемных источников тепла с бо́льшим экспоненциальньм порядком по параметру $\varepsilon$ при $\varepsilon \rightarrow 0$, чем в рассматриваемой задаче.

ЗАмечание 2. Сильную неоднородность задаче (7) придает сильная неоднородность границы области $\Omega_{\varepsilon}$ (за счет большого количества полостей в $\Omega_{\varepsilon}$ при $\varepsilon \rightarrow 0)$, а также быстрая осцилляция при $\varepsilon \rightarrow 0$ коэффициента $a_{\varepsilon}(x)$ из закона Ньютона, по которому идет теплообмен на границе полостей. Но можно усилить неоднородность задачи (7), если рассматривать тело с тензором теплопроводности, имеюшим $\varepsilon$-периодические коэффициенты $K_{i j}^{\varepsilon}(x)=K_{i j}(x / \varepsilon)$, где $K_{i j}(y)-$ гладкие 1-периодические по всем переменньм функции. Результаты теорем 1 и 2 обобшаются и на этот случай. 


\section{Список литературы}

1. Бахвалов Н. С., Панасенко Г. П. Осреднение процессов в периодических средах. М.: Наука, 1984.

2. Олейник О.А., Иосифьян Г. А., Шамаев А. С. Математические задачи теории сильно неоднородных сред. М.: Изд-во МГУ, 1990.

3. Жиков В. В., Козлов С. М., Олейник О. А. Усреднение дифференциальных операторов. М.: Наука, 1993.

4. Сукретньй В. И. Усреднение краевых задач для эллиптических уравнений в перфорированных областях // УМН. 1983. Т. 38. №6. С. 125-126.

5. Сукретный В. И. Асимптотическое разложение решений третьей краевой задачи для эллиптических уравнений второго порядка в перфорированных областях // УМН. 1984. T. 39. № 4. С. 120-121.

6. Сукретный В. И. О задаче стационарной теплопроводности в перфорированных областях // Препринт 84.48. Киев: Институт математики АН УССР, 1984.

7. Пастухова C. E. Метод компенсированной компактности Тартара в усреднении спектра смешанной задачи для эллиптического уравнения в перфорированной области с третьим краевьм условием // Матем. сб. 1995. Т. 186. № 5. С. 127-144.

8. Пастухова C.E. О погрешности усреднения для задачи Стеклова в перфорированной области // Дифференг. уравнения. 1995. Т. 31. №6. С. 1042-1054.

Московский институт

Поступила в редакцию

радиотехники, электроники, автоматики

27.06 .1995 